\title{
Evaluación de la estructura del componente regular de los programas departamentales de hábitos y estilos de vida saludable, Colombia, 2014-2015*
}

\author{
Evaluation of the structure of the regular component of the departmental programs of habits and healthy lifestyles, \\ Colombia, 2014-2015
}

Avaliação da estrutura do componente regular dos programas de hábitos e estilos de vida departamentais, Colômbia, 2014-2015

\author{
Oscar Ricardo Torres Moya ${ }^{\text {a }}$ \\ Escuela de Cadetes de Policía General Francisco de Paula \\ Santander, Colombia \\ ORCID: http://orcid.org/0000-0003-4362-4852 \\ Franklyn Edwin Prieto Alvarado \\ Universidad del Rosario, Colombia \\ Instituto Nacional de Salud, Colombia \\ ORCID: http://orcid.org/0000-0002-2079-7251
}

Fecha de recepción: 16 Mayo 2017

DOI: https://doi.org/10.11144/Javeriana.rgps17-34.esrc

Resumen:

Los programas comunitarios de actividad física son una estrategia prometedora para combatir el sedentarismo. En Colombia existe el Programa Nacional de Hábitos y Estilos de Vida Saludable. Establecer la estructura en su propósito de evaluación general es fundamental para conocer su relevancia y coherencia. El objetivo del estudio fue evaluar la estructura y la variabilidad del componente regular de los programas departamentales de hábitos y estilos de vida saludable, Colombia 2014-2015. La investigación contó con un análisis documental, una valoración de los modelos lógicos y una entrevista semiestructurada. La racionalidad presentó diferencias en lo que se refiere al diagnóstico y se encontró consenso en el establecimiento de objetivos; estos responden a las necesidades y problemas de la población y son tratados a través de estrategias que varían muy poco. Las metas que sugieren los programas, en próximos desarrollos, deberán tener en cuenta las conductas y el contexto de los usuarios.

Palabras clave: evaluación de programas, actividad física, promoción de la salud, campañas de salud, salud pública, prevención de enfermedades.

\begin{abstract}
:
Community programs of physical training are a promising strategy to struggle against sedentariness. In Colombia, there is a National Program for Healthy Habits and Lifestyle. To ascentain the program structure from its general evaluation purpose becomes essential to know its relevance and coherence. This study aims to evaluate the structure and variability of the regular component of the Province Programs for Healthy Habits and Lifestyle, Colombia 2014-2015. This research consisted of a documentary analysis, an assessment of logical models, and a semi-structured interview. The logic processing of the data showed differences regarding the diagnosis and a consensus was found regarding the setting out of objectives, which are set in reply to the needs and problems of the population and are addressed through hardly variable strategies. The goals proposed in the programs, for future developments, should take into account the user's behavior and context.

Keywords: program evaluation, physical training, health promotion, health campaign, public health, disease prevention.

\section{Resumo:}

Os programas comunitários de atividade física são uma estratégia promissória para combater o sedentarismo. Na Colombia existe o Programa Nacional de Hábitos e Estilos de Vida Saudável. Estabelecer a estrutura no seu propósito de avaliação geral é fundamental para conhecer sua relevância e coerência. O objetivo do estudo foi avaliar a estrutura e a variabilidade do componente regular dos programas departamentais de hábitos e estilos de vida saudável, Colômbia 2014-2015. A pesquisa incluiu uma análise documental, uma valoração dos modelos lógicos e uma entrevista semiestruturada. A racionalidade apresentou diferenças no que se refere ao diagnóstico e encontrou consenso no estabelecimento de objetivos; eles respondem às necessidades e problemas da população e são tratados através de estratégias que variam muito pouco. As metas sugeridas pelos programas, em próximos desenvolvimentos, deverão levar em conta os comportamentos e contexto dos usuários.
\end{abstract}


Palavras-chave: avaliação de programas, atividade física, promoção da saúde, campanhas de saúde, saúde pública, prevenção de doenças.

\section{Introducción}

Los programas comunitarios de actividad física son considerados una estrategia prometedora para combatir el sedentarismo, son gratuitos, se desarrollan en escenarios públicos de diferentes tipos, para la práctica de diferentes actividades (ejercicios corporales, aeróbico, musicalizado, juego estructurado, etc.), cuentan con la guía de un entrenador o educador físico y facilitan el acceso a personas en estado de vulnerabilidad como mujeres, adultos mayores y personas de estratos socioeconómicos bajos [1,2,3].

Los ministerios de Salud y Protección Social, de Educación Nacional y de Cultura y Coldeportes "se comprometen a buscar los mecanismos para la inclusión de la promoción de la actividad física en las agendas públicas, el establecimiento de planes, programas y proyectos, y su sostenibilidad técnica y financiera, así como la evaluación del impacto de las intervenciones y el apoyo a los procesos de investigación” [4]. Esto, de conformidad con las recomendaciones de la Estrategia Mundial sobre Régimen Alimentario y Salud de la OMS [5], que buscan un impacto sobre las enfermedades no transmisibles y la obesidad/sobrepeso [6].

A partir de este compromiso se estructura el programa Hábitos y Estilos de Vida Saludable, que se construye desde el nivel nacional y es transferido secuencialmente a los niveles departamentales y municipales, de acuerdo con la situación epidemiológica nacional, con la intención de ajuste en los niveles locales. Cada departamento puede adaptarlo a su contexto cultural y ambiental. El programa es liderado en el nivel departamental por una institución pública responsable del deporte, la recreación y la actividad física, mediante un gestor o coordinador, licenciado en educación física [6].

El programa en su componente regular se ofrece directamente a las comunidades (barrios, grupos, centros urbanos, etcétera) y se desarrolla con un mínimo de tres sesiones de sesenta minutos por grupo, realizando actividades propias al macro y micro entorno de cada población, donde sobresalen rumba aeróbica, caminatas y gimnasia aeróbica, entre otras [7]. Esta situación favorece que el programa tenga variabilidad, porque no se hacen las mismas actividades en todos los departamentos.

La estructura de un programa tiene aspectos como la definición de una meta, con lenguaje simple y claro; la formulación de objetivos con características de especificidad, medibles, alcanzables, realistas y con un plazo determinado; identifica y califica factores contribuyentes con evidencia científica; diseña la intervención, determinando la estrategia de salud; investiga las intervenciones basadas en la evidencia, compara y selecciona la intervención; formula objetivos de proceso y desarrolla un plan de implementación. Estos aspectos deben ser evaluados continuamente para fortalecer la estructura y el desarrollo normal del programa [8].

Establecer la estructura de los programas a partir de la coherencia (adecuación de la jerarquía de objetivos establecidos en el programa) y la racionalidad, que implica el análisis de la relevancia (diagnóstico plasmado en el programa) y pertinencia (calidad y adecuación de la definición de los objetivos), permitirá la revisión de los elementos que justifican los programas de actividad física comunitaria y su necesidad y la forma como se articulan con otras políticas o programas establecidos [9].

Los programas deben ser evaluados, para lo cual hay diferentes metodologías [10], entre las que se encuentran las de análisis de contenido que han sido empleadas para la observación de políticas públicas y que el Ministerio de Salud y Protección Social ha utilizado como método de evaluación $[9,11,12]$.

Osuna propone este tipo de evaluación como:

[...] alternativa a la concepción tradicional de la evaluación como última etapa del proceso de planificación, optamos por una visión integral donde la evaluación se realiza en todas las etapas del diseño de programas, analizando la pertinencia y coherencia de los problemas, objetivos e instrumentos planteados, el proceso de aplicación del programa y sus resultados. 
Uno de los objetivos de la evaluación es proporcionar retroalimentación para el proceso durante el desarrollo de este [13].

\section{Pregunta de investigación}

¿Cuál es la estructura y la variabilidad del componente regular de los programas departamentales de hábitos y estilos de vida saludable, Colombia 2014-2015?

\section{Objetivo general}

Evaluar la estructura y la variabilidad del componente regular de los programas departamentales de hábitos y estilos de vida saludable, Colombia 2014-2015.

\section{Objetivos específicos}

- Identificar la racionalidad de los programas de hábitos y estilos de vida saludable de la vigencia 2014-2015.

- Identificar la coherencia de los programas de hábitos y estilos de vida saludable de la vigencia 20142015.

\section{Método}

La metodología de investigación tuvo varios componentes. El primero, un análisis documental, en el que se utilizó como referencia la técnica de revisión sistemática para opiniones, documentos y experiencias del Instituto Joanna Briggs [14] — matriz de requisitos mínimos que debía tener un programa para posteriormente ser analizado- y la Matriz de Resumen de Evaluación de Estructura, basada en el Consejo Nacional de Evaluación de la Política de Desarrollo Social (Coneval) de México. [15] El segundo, la valoración de los modelos lógicos, construidos en cada uno de los programas departamentales, utilizando la matriz propuesta por el Department for International Development del Reino Unido [16]. Finalmente, una entrevista semiestructurada para verificar lo encontrado en las dos fuentes anteriores.

La población de estudio estuvo conformada por los programas departamentales. El soporte fue el documento que Coldeportes exige a los departamentos como requisito para el convenio que establece con la entidad territorial y que tiene como nombre Proyecto de Hábitos y Estilos de Vida Saludables vigencia 2014 y 2015 [17,18]. Se excluyeron Bogotá y Antioquia por ser programas con características diferentes (iniciaron antes del programa nacional).

Se tomó como criterio de exclusión no cumplir con los requisitos de la matriz de chequeo. Este instrumento fue modificado de la metodología del Instituto Joanna Briggs [14]. Esta matriz incluía cinco criterios mínimos de inclusión y diez que determinan la integridad del documento. Los programas de la vigencia 2014 (22 documentos) y los de 2015 (19 documentos) cumplieron con los criterios. No se presentaron exclusiones.

\section{Análisis documental}

Las categorías para la evaluación de diseño o estructura son racionalidad y coherencia. La primera hace referencia a la evaluación de las relaciones entre las necesidades, los problemas y los objetivos. Para su estudio se 
divide en dos conceptos: la relevancia, que es la evaluación del diagnóstico para el programa, y la pertinencia, que es la evaluación de la calidad y la adecuación de la definición de los objetivos [11,12].

La segunda, coherencia —evaluación de la adecuación de la jerarquía de objetivos establecidos en el programa- puede ser interna o externa. La interna es la evaluación de la articulación de los objetivos con los instrumentos de la estrategia y su adecuación con los problemas, y la externa, el análisis de la compatibilidad tanto de los objetivos como de la estrategia con otras políticas y programas con los que puedan tener sinergias o complementariedad en el tiempo o en el espacio [12].

Los documentos fueron fotografiados en el archivo de Coldeportes, que autorizó una copia fotográfica. Posteriormente, se les aplicó la matriz de chequeo adaptada del Instituto Joanna Briggs [14]. Debido a que todos los documentos aprobaron los requisitos mínimos de esta matriz, a continuación se aplicó la adaptación de la Matriz de Resumen de Evaluación de Estructura de Coneval [15], cuyo instrumento se fundamenta en la aplicación de seis dominios: planteamiento del problema (cuatro), descripción del programa (cuatro), relevancia (ocho), pertinencia (seis), coherencia interna (cinco) y coherencia externa (cinco). El instrumento proporciona una calificación por dominio de desempeño. El instrumento tuvo evaluación de apariencia y contenido por expertos.

\section{Análisis del modelo lógico}

Un modelo lógico es una manera de presentar un programa de forma gráfica y sistemática, en el cual se presenta la relación entre las actividades y los efectos esperados. Además, es una herramienta que facilita la planificación, la implementación y la evaluación exitosa de los programas [19].

Los modelos lógicos tienen cuatro componentes: qué necesita el programa, qué hace el programa, quién o qué cambiará por el programa y los factores externos que influyen en el logro de los resultados. Estos componentes fueron analizados en cada modelo. A cada documento de programa se le realizó la evaluación de los modelos lógicos, para lo cual se utilizó la herramienta del Departament for International Development de Reino Unido [16].

\section{Entrevista semiestructurada}

La información fue complementada mediante una entrevista semiestructurada dirigida a los gestores (encargados directos de los programas departamentales). Se opta por el tipo de metodología semiestructurada pues se conocen las categorías, pero se requiere precisar acepciones u obtener mayor información sobre categorías emergentes. El guion estuvo compuesto por trece preguntas en tres componentes: hechos relevantes del planteamiento (cuatro), de la descripción (cinco) y de la operación (cuatro). Se emplearon todas las recomendaciones que hay para desarrollar una adecuada entrevista semiestructurada [20].

Las entrevistas se realizaron en el marco del Encuentro Nacional de Programas de Hábitos y Estilos de Vida Saludable, Festival Audiovisual y del Cuento Hábitos y Estilos de Vida Saludable 2015, desarrollado en Nilo, Cundinamarca entre el 9 y el 13 de diciembre de 2015. Al encuentro asistieron los veintidós gestores de los departamentos de la vigencia 2014-2015.

Los gestores fueron distribuidos en cuatro grupos, conformados por un entrevistador y un relator que registró el ambiente de la entrevista y su grabación en audio. Los entrevistadores fueron los estudiantes de la Maestría de Actividad Física y Salud de la Universidad del Rosario, IV cohorte, pertenecientes al macroproyecto de investigación, quienes tuvieron entrenamiento y ensayos conductuales de las entrevistas y conocieron el guion y el consentimiento informado de estas. 
El ejercicio se desarrolló durante los cinco días que duró el encuentro. Después de realizar dos entrevistas por grupo, se reunían los entrevistadores y se determinaba la saturación de la información y el establecimiento de categorías emergentes. Se realizaron veintidós entrevistas a los gestores participantes.

Se transcribieron las entrevistas, digitándolas a medida que se escuchaban, así como las bitácoras y las observaciones generales en el momento de la entrevista. Se desarrolló una verificación de que el material estuviera completo y con la calidad necesaria para ser analizado. Para obtener un panorama del material se construyen matrices, a partir de las categorías de análisis (racionalidad, coherencia, hechos relevantes del planteamiento del problema, de la descripción y de la operación del programa), y se codifican de acuerdo con estas. Este análisis fue desarrollado a partir de lo presentado por la metodología de análisis de contenido en la entrevista semiestructurada $[20,21]$.

En cuanto al rigor y la calidad científica de la metodología cualitativa, la realización de la investigación se basó en la propuesta desarrollada por Castillo et al. [22], quienes presentan tres criterios en la construcción de la calidad de este tipo de investigaciones.

La credibilidad se logra porque los hallazgos son asumidos como reales o verdaderos por las personas que están en contacto con el fenómeno estudiado, por esta razón se entrevistó a los gestores departamentales, que son quienes conocen desde la estructura a los programas, y se analizaron los programas de hábitos y etilos de vida saludable, así como documentos primarios suministrados por cada uno de los departamentos. El análisis de los modelos lógicos se realizó con base en un instrumento.

La auditabilidad o confirmabilidad, que hace referencia a la neutralidad de la interpretación de los resultados, se logra cuando otro investigador puede "seguir la pista". Por ello, todas las entrevistas fueron grabadas, y la revisión documental, así como el análisis de los modelos lógicos, se realizó a través del mismo protocolo (uno para la revisión documental y otro para los modelos lógicos). La transferibilidad, es decir, la posibilidad de transferir los resultados a otros grupos o contextos, es lo que se espera a mediano o largo plazo con el desarrollo de esta investigación [22]. Todas las entrevistas respondieron al protocolo establecido para tal fin, basado principalmente en el presentado por María J. Mayan [20].

El estudio fue aprobado por el Comité de Ética del Instituto Nacional de Salud Pública de México y de la Universidad del Rosario. Las entrevistas desarrolladas fueron antecedidas por una explicación al entrevistado acerca del objetivo y los alcances de la investigación, así como de la lectura del consentimiento informado.

\section{Resultados}

\section{Análisis documental}

Todos los programas departamentales de las vigencias analizadas cumplieron con la lista de chequeo para ser incluidos en el estudio. Tres departamentos que presentaron programa en la vigencia 2014 no lo hicieron en el 2015: Cundinamarca, Guaviare y Cesar. Los demás departamentos que presentaron programa en las dos vigencias y a los cuales se les realizó el análisis documental fueron: Arauca, Atlántico, Bolívar, Boyacá, Caldas, Caquetá, Casanare, Cauca, Córdoba, Cundinamarca, Guainía, La Guajira, Guaviare, Huila, Nariño, Norte de Santander, Putumayo, Quindío, Risaralda, Santander, Sucre y Tolima. Se aclara que los departamentos faltantes del territorio nacional, exceptuando Bogotá y Antioquia (no se tuvieron en cuenta por razones ya enunciadas en el método), no tienen el programa de actividad física objetivo de esta investigación.

No se encontraron diferencias importantes de los programas de los departamentos de 2014 y 2015, y no se evidenciaron cambios en el diagnóstico, el problema y el objetivo (racionalidad), mientras que en las metas y en las actividades (coherencia) se presentaron diferencias en las metas de cobertura mediadas por la capacidad instalada de cada uno de los departamentos. 
Para la relevancia se evidenció lo que se presenta en la figura 1. La problemática en los diferentes programas se puede clasificar en tres. Arauca, Casanare, Cauca, La Guajira, Guaviare, Nariño y Sucre lo enuncian desde un contexto mundial, mientras Atlántico, Caldas, Boyacá, Cundinamarca, Huila y Norte de Santander lo particularizan a las regiones. Hay enunciados generales y focalizados a la población particular en Bolívar, Putumayo, Guainía, Quindío, Tolima Córdoba, Caquetá, Risaralda y Santander.

Para el diagnóstico de situación se utilizan estudios de la OMS, la Encuesta Nacional de la Situación Nutricional de Colombia 2010 [23] y estadísticas generales de la Nación. Aquellos que caracterizan la población, se remiten a estudios de entidades regionales para documentar su diagnóstico. La aplicación de la matriz Coneval [18] muestra que la justificación teórica o empírica documentada que sustente el tipo de intervenciones mínima sucedió en tres departamentos (Córdoba, Guainía y Santander).

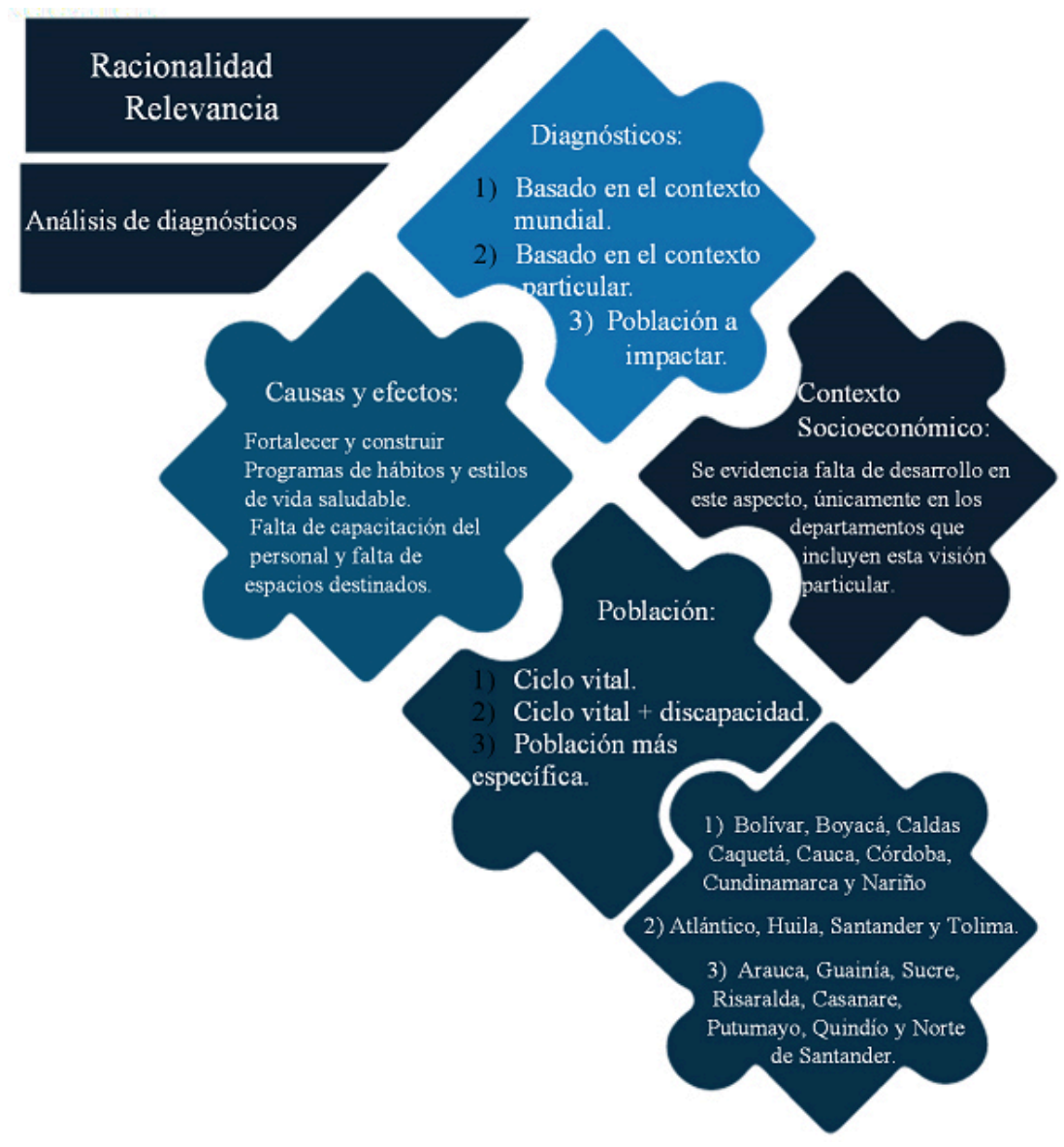

FIGURA 1

Resultado de la relevancia de los programas departamentales de hábitos y estilos de vida saludable

La población objetivo que enuncian los programas se puede enmarcar en todo el ciclo vital, pero algunos extienden su cobertura a grupos especiales, como población privada de la libertad o enfermos crónicos, entre otros. En la relevancia de los programas se presenta y analiza de forma poco detallada el contexto socioeconómico de los departamentos.

Sobre la pertinencia, los programas están orientados al mismo objetivo, relacionado con fomentar y fortalecer los hábitos y estilos de vida saludable como elemento protector de salud y de calidad de vida, a través de actividad física, hábitos alimenticios y espacios 100\% libres de humo de tabaco. 
En lo que se refiere al logro que se espera de los programas, este es claro, especificado y controlado por los responsables del programa. Mediante la lectura del objetivo se puede establecer cuáles serán los resultados; sin embargo, los programas no presentan plazo para el logro del objetivo y su medición está basada en indicadores que pueden estar dejando de un lado categorías importantes, por ejemplo, las contextuales, la motivación, la empatía, etcétera. Se asume que la vigencia determina el periodo del objetivo.

\section{Resultados de la coherencia}

\section{Coherencia interna}

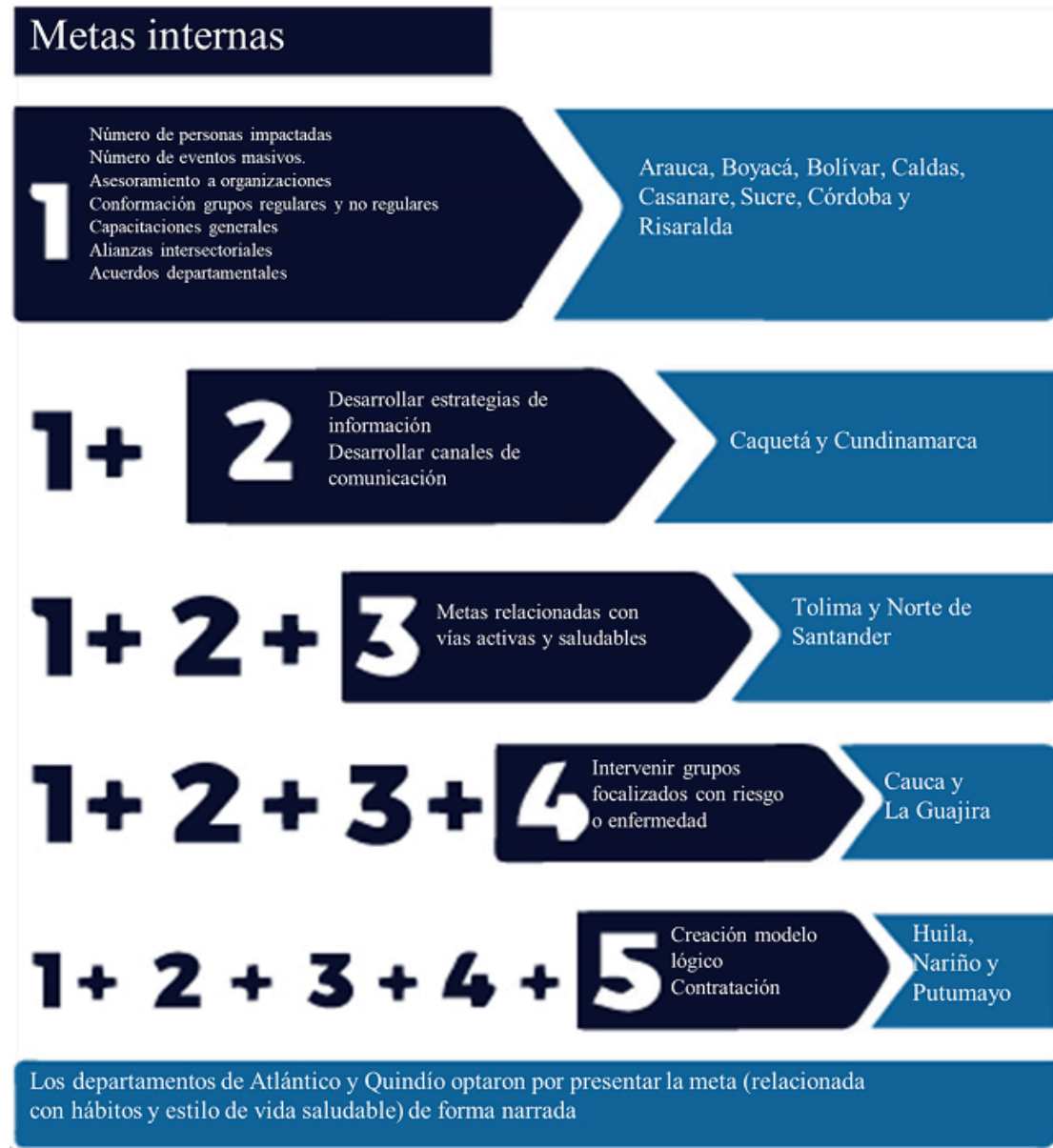

FIGURA 2

Resultados de la coherencia interna. Metas de los diferentes programas departamentales del programa Hábitos y Estilos de Vida Saludable

De acuerdo con el instrumento adaptado de Coneval [18], los departamentos que demuestran un mejor diseño del programa de Hábitos y Estilos de Vida Saludable fueron Santander y Córdoba, en tanto que aquel con menos puntaje es Cesar (tabla 1). 
TABLA 1

Resultado de la evaluación de la estructura del programa Hábitos y Estilos de Vida Saludable, adaptado de Coneval, y valoración de los modelos lógicos a través de la matriz propuesta por el Department for International Development Reino Unido

\begin{tabular}{|c|c|c|c|c|c|c|}
\hline \multirow{2}{*}{ Departame nto } & \multicolumn{3}{|c|}{ Racionalidad } & \multirow{2}{*}{$\begin{array}{l}\text { Coherencia } \\
\text { Interna n/16 }\end{array}$} & \multirow{2}{*}{ Total $n / 40$} & \multirow{2}{*}{$\begin{array}{c}\begin{array}{c}\text { Evaluación } \\
\text { modelo } \\
\text { lógico }\end{array} \\
\text { N } / 26\end{array}$} \\
\hline & $\begin{array}{c}\text { Relevancia } \\
\text { n/8 } \\
\end{array}$ & $\begin{array}{c}\text { Pertinencia } \\
\text { n/16 } \\
\end{array}$ & $\begin{array}{l}\text { Total } \\
\mathbf{n} / \mathbf{2 4} \\
\end{array}$ & & & \\
\hline Arauca & 4 & 13 & 17 & 12 & 29 & 14 \\
\hline Atlántico & 4 & 13 & 17 & 12 & 29 & 15 \\
\hline Bolivar & 6 & 14 & 20 & 11 & 31 & 16 \\
\hline Boyacá & 6 & 14 & 20 & 11 & 31 & 15 \\
\hline Caldas & 4 & 14 & 18 & 11 & 29 & 16 \\
\hline Caquetá & 6 & 14 & 20 & 11 & 31 & 15 \\
\hline Casanare & 4 & 13 & 17 & 11 & 28 & 17 \\
\hline Cauca & 4 & 13 & 17 & 11 & 28 & 18 \\
\hline Córdoba & 7 & 14 & 20 & 11 & 32 & 14 \\
\hline Cundinamarca & 4 & 14 & 18 & 11 & 29 & 15 \\
\hline Guainia & 6 & 13 & 19 & 11 & 30 & 16 \\
\hline Guajira & 4 & 14 & 18 & 11 & 29 & 18 \\
\hline Guaviare & 4 & 13 & 17 & 12 & 29 & 16 \\
\hline Huila & 5 & 15 & 20 & 11 & 31 & 16 \\
\hline $\begin{array}{l}\text { Nariño } \\
\end{array}$ & 4 & 12 & 16 & 13 & 29 & 15 \\
\hline Norte de Santander & 5 & 14 & 19 & 11 & 30 & 13 \\
\hline Putumayo & 4 & 14 & 18 & 11 & 29 & 18 \\
\hline Quindio & 5 & 14 & 19 & 11 & 30 & 15 \\
\hline Ris aralda & 4 & 13 & 17 & 11 & 28 & 14 \\
\hline Santander & 7 & 14 & 21 & 11 & 32 & 16 \\
\hline Sucre & 3 & 14 & 16 & 11 & 27 & 10 \\
\hline Tolima & 4 & 14 & 18 & 11 & 29 & 16 \\
\hline
\end{tabular}

En lo que se refiere a la coherencia externa, los documentos analizados no permitieron evidenciar las sinergias con otros programas o instituciones, que son mencionadas, pero requieren mayor desarrollo.

\section{Análisis de los modelos lógicos}

Dentro de los cuatro componentes que tienen los modelos lógicos se encontró que tres departamentos presentan factores externos que influyen en la realización del programa.

La mayor claridad se encuentra en las actividades que hacen; todos los departamentos lo ilustran en sus modelos, mientras en lo referente a resultados e impacto, exceptuando los departamentos que presenta la tabla, no presentan indicadores sino resultados a corto plazo, y en lo que se refiere al impacto lo presentan como resultado a largo plazo. 
TABLA 2

Resumen de los modelos lógicos de los programas departamentales de hábitos y estilos de vida saludable

\begin{tabular}{|c|c|c|c|}
\hline \multicolumn{4}{|c|}{ Aspectos centrales que ilustra el mode lo lógico en cada programa } \\
\hline qué necesita el programa & qué hace el programa & $\begin{array}{c}\text { quién o qué cambiará } \\
\text { del programa }\end{array}$ & factores externos \\
\hline Norte de Santander & & Cauca & Arauca \\
\hline Atlántico & & Putumayo & Boyacá \\
\hline Sucre & & La Guajira & Casanare \\
\hline & Todos los programas & Estos departamentos & Then \\
\hline $\begin{array}{l}\text { Estos departamentos no } \\
\text { presentan lo que necesitan } \\
\text { (entradas) Los demás } \\
\text { departamentos la hacen }\end{array}$ & $\begin{array}{l}\text { presentan en el modelo, } \\
\text { lo que hacen. }\end{array}$ & $\begin{array}{l}\text { dan la diferencia al } \\
\text { presentar indicadores. } \\
\text { Los demás } \\
\text { departamentos no b } \\
\text { realizan. }\end{array}$ & $\begin{array}{l}\text { Estos departamentos hacen la } \\
\text { diferencia al presentar estos } \\
\text { factores. Los restantes } \\
\text { departamentos no lo hacen. }\end{array}$ \\
\hline
\end{tabular}

La matriz utilizada demuestra que Putumayo resume de mejor manera su programa implementado y Sucre requiere una mayor explicación.

\section{Entrevista semiestructurada}

En el análisis del diagnóstico sobresale la necesidad que tuvieron los gestores de tener información local de las enfermedades crónicas y los hábitos y estilos de vida saludable en general, pues algunos aseguran que antes del programa no se tenía información. Aseveraciones de los gestores de Putumayo, Guainía y Cundinamarca y Tolima lo confirman:

"No había un programa de actividad física instaurado como tal, había programas de diseño deportivo. Entonces, solamente se asistía las poblaciones de los ocho a los quince años, pero eran las clases de educación física, entonces por eso decidió llevar este programa al de Hábitos y Estilos de Vida Saludable al departamento precisamente porque no todas las poblaciones se estaban viendo afectadas. (Gestor del programa en Guainía)"

"En el 2011 el Instituto Nacional de Salud realizó una investigación sobre la carga de enfermedades crónicas no transmisibles en el departamento de Putumayo, arrojó pues una información que fue útil para dar un diagnóstico, una línea de base para estructurar el proyecto en todo Coldeportes nacional. (Gestor de Putumayo)"

"Siempre tomamos los lineamientos que Coldeportes está dando y las diferentes directrices, luego partimos a hacer una caracterización de los municipios que realmente tienen el potencial para tener el programa en su municipio, valga la redundancia, desde ahí que es lo que tenemos en cuenta, la población, tipo de población, necesidades que tiene cada uno de los municipios, grandes municipios son los que estamos impactando, entonces revisamos que ha habido antes de arrancar el programa, que fue lo que tuvimos antes, que fue lo que logramos, que dificultades hubo, que no se logró y que si se logró y desde ahí podemos construir las diferentes estrategias y objetivos que queremos lograr en esos municipios, desde ahí ya podemos escoger los municipios impactados. (Gestor de Cundinamarca)"

"Identificamos de acuerdo al último informe epidemiológico del departamento entonces sabíamos datos estadísticos de cuantas personas fallecen por ECNT de acuerdo a la diabetes, a la hipertensión, enfermedades cardiovasculares o accidentes cardiovasculares, y esta información la revisábamos en los planes de desarrollo que tenía cada departamento o el departamento del Tolima y solicitábamos a la Secretaría de Salud departamental que nos diera el informe actualizado de mortalidad y morbilidad por ECNT. A partir de ahí hacíamos un análisis del comportamiento de la comunidad desde la inactividad física y esto lo hicimos investigando los planes de desarrollo que tienen los municipios donde íbamos a impactar, a partir de ahí identificamos que el departamento del Tolima su problemática es la falta de programas efectivos. (Gestora del Tolima)"

"La población que atiende el programa, según las personas entrevistadas del programa de Hábitos y Estilos de Vida Saludable, no está determinada en una población en especial. Al contrario, el programa es muy abierto y en determinadas situaciones se atiende población particular, como lo mencionan los siguientes gestores."

"Para toda la comunidad caldense, buscamos sí que haya una prioridad en cierta población vulnerable; estamos apuntando a lo que son los desplazados, los indígenas, los afrocaldenses. El departamento es muy rico culturalmente, entonces buscamos que el programa vaya más a las personas que necesitan otras posibilidades de libre esparcimiento y obviamente también 
atendiendo a la necesidad de disminuir los índices de sedentarismo y los grados de obesidad que hay en el departamento. (Gestora de Caldas)"

"No, el programa no está diseñado para alguna población específica, ni siquiera caracterizada, es un programa que trabaja con todo el ciclo vital. Es decir, desde el niño de cero años hasta la persona mayor que tiene más de 64 años hay que hacer una caracterización de ellos y se debe tener en cuenta al momento de hacer la sesión, [...] es un programa muy abierto. (Gestor del Quindío)"

"El programa está diseñado para impactar desde los niños de primera infancia hasta el adulto mayor y también inclusión de personas con diferentes discapacidades, pero pues la mayor población que asiste es entre jóvenes adultos y adultos mayores es la mayor población que atiende el programa actualmente. (Gestor del Caquetá)"

"A toda la población, no hay ninguna cosa que diga no puede hacer, es más si detectamos una discapacidad a esa persona también se invita a que haga actividad física, allá van con su silla de ruedas o con sus muletas y trabajan, porque es que esto no es solamente tiene que ver con la actividad física, sino que ha integrado a la población. (Gestor del Atlántico)”

En relación con el análisis de la pertinencia del programa de Hábitos y Estilos de Vida Saludable, las personas entrevistadas enfocaron la respuesta del objetivo del programa a bajar la prevalencia del sedentarismo mediante la actividad física, sin olvidar la alimentación saludable y la protección de espacios libres de humo de cigarrillo y mejorar la calidad de vida de las personas que asisten al programa:

"El objetivo es promover lo que es actividad física, alimentación saludable y promover espacios cien por ciento libres de humo de tabaco, al igual que el no consumo de alcohol. (Gestor del Cauca)"

"El objetivo principal del programa es mejorar la calidad de vida de los habitantes de Nariño, ¿cómo?, generando cambios comportamentales, permitiéndoles alternativas de actividad física para que ellos de acuerdo a los horarios que tengan de trabajo puedan acceder a nuestros grupos, nosotros no trabajamos en sitios cerrados, tratamos de trabajar en parques para que la población sea mucho mayor, para que la gente por medio de la observación diga: [...] que chévere esto a la próxima vengo. (Gestor de Nariño)"

"El objetivo del programa básicamente es aumentar la prevalencia de la práctica de la actividad física en el departamento, o sea que las personas practiquen más, [...] básicamente traducido a palabras muy comunes es eso que la gente se enamore de la actividad física que la gente viva la actividad física, que la gente sea más activa y más saludable. (Gestor de Cundinamarca)”

Las metas que comunican los entrevistados refieren resultados y están mediadas por la capacidad instalada que tiene cada departamento, como es el caso de Risaralda, Tolima y Huila:

"Pues en el proyecto 2015 se hablaba de aumentar el impacto por cantidad de personas, entonces el año pasado había 1900 usuarios regulares registrados, ya este año se aumentó a 2300 y la idea es seguir aumentando. Generalmente, estos objetivos se traducen en cantidad de personas impactadas, cobertura o porcentajes; pero pienso que se debería ver realmente cuál es el impacto en salud que tienen los usuarios, o sea, si la persona está mejorando su presión arterial, si está bajando su índice de masa corporal o está bajo la normalidad, que haya un seguimiento en general. (Gestor de Risaralda)”

"[...] Las metas van acorde al número de monitores, al número de acciones, entonces nosotros si tenemos cinco monitores cada monitor debe conformar cinco o seis grupos regulares [...] Entonces de acuerdo al número de monitores, así mismo cuantos grupos regulares como mínimo, cuantas personas como mínimo, cuantas asesorías y eventos masivos, cada mes hay que hacer un evento. (Gestor de Boyacá)"

"Aumentar las cifras en participación en AF, la adopción la meta es la adopción de |aumentar esa cifra de la opción de conductas saludables. (Gestor de Huila)"

En cuanto a la coherencia (interna y externa), los entrevistados resaltan la importancia de utilizar metodologías acordes a la población y el contexto en general. Así lo mencionan los gestores de de Bolívar, Atlántico y Guainía:

“[...] en la costa la gente le gusta el baile y eso ha sido como un imán para arrastrar a personas porque ha sido bien difícil llevarlos a una máquina, a que hagan cosas verdad, y entre otras cosas con esta metodología que utilizamos de la Actividad Física musicalizadas es poco lo que necesitan, la persona puede ir con o sin uniforme puede ir con tenis o sin ellos llegan allá como si fuesen a sus labores de campesino. (Gestor del Atlántico)"

"Actividad física musicalizada, también es algo importante que hemos trabajado con nuestro folclor. Porque no podemos quitarles ese sentir a las personas, porque les gusta bailar su cumbia, el merecumbé y nosotros no vamos a ponerles música que no los motiva. Entonces este factor de meterle este folclor a los grupos regulares a nosotros nos ha dado buenos resultados. (Gestor de Bolívar)" 
"Se dejan de hacer por momentos las clases musicalizadas, o sea con el sonido, pero, sin embargo, qué se acoge, se acogen los buenos calentamientos la buena movilidad articular, sí, la fase central se hacen juegos estructurados y la fase final [...] estiramiento. Se mantienen los lineamientos de las sesiones de actividad física, pero [...] se les vende con juegos estructurados sin música o la música puede ir de fondo, la mayoría de los grupos hay que hacerlo así. La visita que nos hizo Adriana Almanza nos dijo, no todo puede ser musicalizado, pues hay grupos que tienen otra necesidad, por ejemplo, los niños con discapacidad, entonces hay que comenzar el trabajo de lateralidad de reconocimiento corporal, etc. (Gestor de Guainía)"

"Novedosos son los eventos, ya te lo dije, los eventos masivos, los hacemos muy culturales, culturales es por decir la celebración del día mundial de la actividad física los usuarios utilizaron una indumentaria propia de la región pollera, música autóctona de la región, eso les genera algún punto como que único al programa, el que a nivel departamental utilicemos esas estrategias. En la costa se ve muchas músicas diferentes: champeta, reggaetón, esa utilización de ese tipo de música dada en nuestro contexto está dándonos un punto favorable, y el carisma de los monitores para llegarle a los usuarios es otro punto favorable para nuestro programa, total. (Gestor de Sucre)"

En la coherencia externa los gestores resaltan la importancia del trabajo intersectorial, sin embargo, algunos tienen sus reparos a la hora de desarrollarlo, como es el caso de los departamentos de Arauca, La Guajira, Huila, Risaralda y Santander:

“[...] El trabajo interinstitucional que se hace con las empresas, las entidades, las organizaciones y donde buscamos ese recurso o aunar esfuerzos para que no seamos solo el programa de Hábitos que realice la actividad, sino que con ayuda de las demás entidades hagamos un [...] coaccionemos las acciones y sean más visibles para la comunidad, como dicen por ahí —una sola golondrina no hace milagros - todo esto dentro del componente regular y los grupos regulares. (Gestor de Arauca)"

"Bueno nuestra estructura se basa en las políticas de Coldeportes, lo único que cambia es con las formas de realizar asesorías institucionales, pero creo que hay mucha congruencia con la política pública nacional. Con relación a la departamental, es un proceso que viene cogiendo fuerza, apenas estamos haciendo un trabajo intersectorial en su primera fase. Pero es un avance en cuanto a política pública poder encontrarnos las instituciones y decir en que nos podemos apoyar. (Gestor de La Guajira)"

"Se creó por ordenanza, no por decreto, el comité departamental intersectorial para la promoción de Hábitos y Estilos de Vida Saludable, donde esta hace parte de ese comité educación, cultura, salud, todas las secretarias hacen parte el SENA, hace parte el ente de educación superior que en este momento es la Universidad Surcolombiana, está la Policía Nacional, está el ejército, entonces, ahí hay un comité departamental grande que digamos que todas las políticas se generan desde allí nosotros tenemos reuniones periódicas y a partir de las necesidades, y pues el tiempo no y los recursos pues nosotros proyectamos las actividades y Secretaría de Educación obviamente está presta a este tipo de actividades. (Gestor del Huila)"

"Traducir esa política al departamento ha sido un poco complicado, ha sido de pronto una materia bien difícil, ya que se manejan por separado la parte de salud y la parte deportiva, entonces lograr esa decisión de salud y a nivel deportivo en el departamento ha sido bastante complicado, pero ya se han logrado más cercanías y más cosas para mirar qué se puede realizar a nivel interinstitucional, pero si es complicado a nivel departamental a algunos lineamientos a nivel nacional. No hay una política definida que ellos digan sí, ya tenemos una política deportiva definida en el departamento, no la hay, no ha sido formulada por parte de gobernaciones, alcaldías, no. Sí, porque allá se trabaja por su lado entonces cuando dicen, por ejemplo, necesitamos mostrar la semana de hábitos entonces aparece todo lo que se está haciendo a nivel de región, pero no se está como igualando a los lineamientos a nivel nacional, cada uno lo está haciendo por su lado. (Gestor de Santander)”

"No, realmente no encuentro nada que yo diga no se debe hacer, antes por el contrario, yo siempre he sido una de las convencidas de que es uno de los mejores programas de Coldeportes, porque es un programa que tiene mucho impacto y como monitora me di cuenta que a las personas le gusta mucho lo que se hace, pero como gestora he tenido problemas más que todo con el trabajo intersectorial. Entonces, se debería tener una mejor guía de cómo se debe hacer un trabajo intersectorial, porque muchas veces cuando se hace un trabajo me dicen que la actividad es una opción, pero no es un trabajo intersectorial. (Gestor de Risaralda)"

A través de un análisis de frecuencia, en las entrevistas realizadas a los gestores se establecieron algunas fortalezas y diferencias de los programas que lideran. 


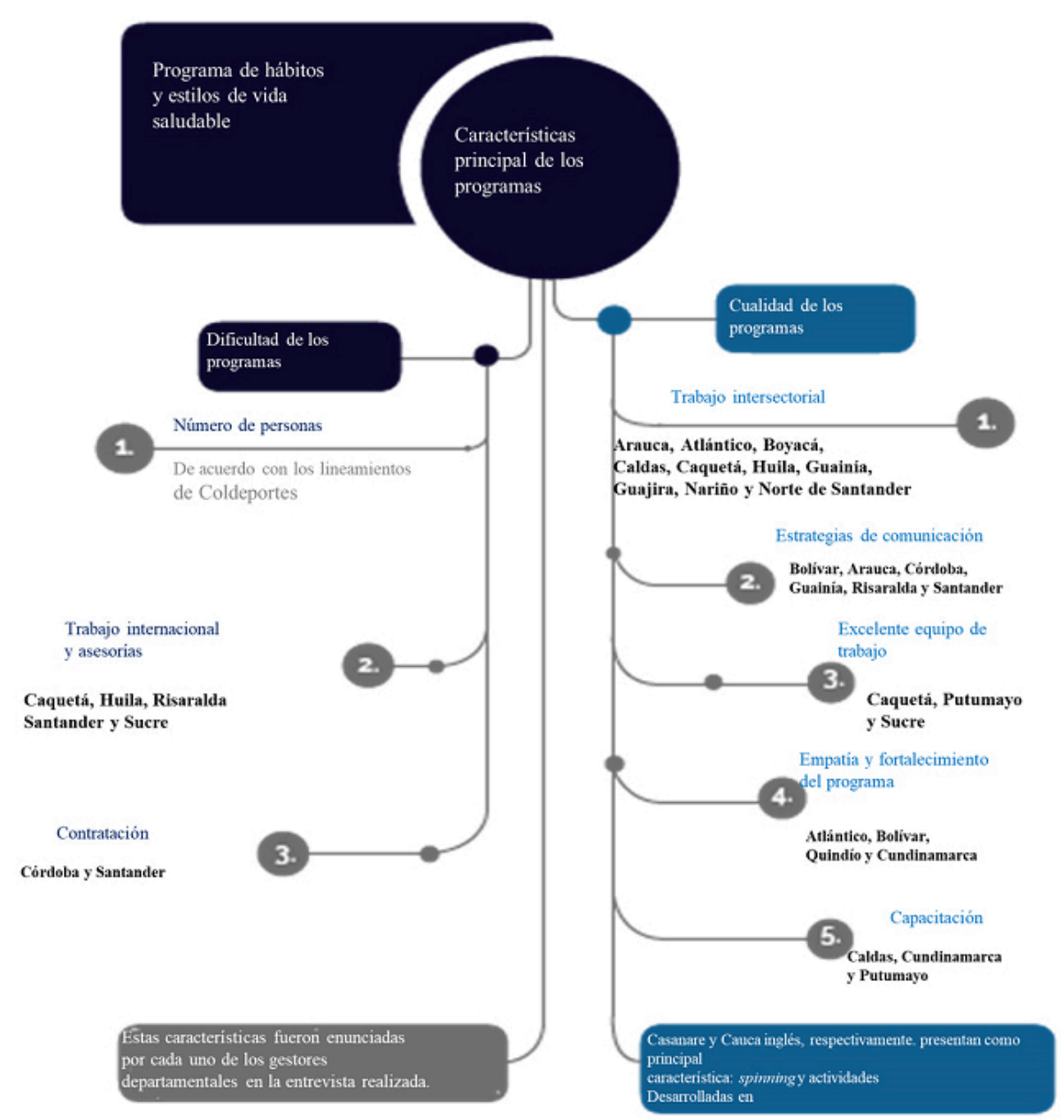

FIGURA 3

Dificultades y principales características de los programas

departamentales de hábitos y estilos de vida saludable.2014-2015

\section{Discusión}

Hay múltiples metodologías para evaluar los programas [10], en el análisis de las políticas públicas se pueden enfocar cuatro dimensiones para ello: el estudio del contenido, el estudio del proceso, los estudios de evaluación de o para las políticas y el estudio del soporte [11]. Esta investigación está orientada a la primera dimensión, y las categorías que se tuvieron en cuenta — racionalidad y coherencia - adquieren una importancia en la evaluación y el análisis en lo que se refiere al contenido, permitiendo constituir cómo se estableció el diagnóstico y su relación con los objetivos y actividades planteadas, además de las sinergias utilizadas con otros programas o políticas orientadas a la misma problemática y teniendo contemplada una metodología para ello $[11,12]$.

Esta evaluación de estructura se realizó en la fase de operación con un enfoque formativo que ayuda a optimizar y encontrar nuevas versiones o reformas al programa en general [23]. La invitación que hace Coneval [15] es a percibir la evaluación desde una concepción holística, desde que se inicia la planificación del programa y no como en una última etapa o fase, independiente y enfocada única y exclusivamente al impacto que ocasiona la intervención en los usuarios, sin dedicar ninguna atención a las acciones que se realizan en la realidad del espacio social del programa, con toda la gama de actores involucrados en este [24]. 
Lo anterior concibe la planificación y la evaluación como procesos relacionados que se orientan en la misma dirección, que tienen como propósito mejorar las políticas y los programas a través de una mejor toma de decisiones, la cual se inicia con una adecuada identificación de la causa y el efecto de los problemas. Contar con un análisis o diagnóstico del problema permite definir con mayor claridad el presupuesto necesario y el modo en el que se van a operar las intervenciones gubernamentales para lograr los resultados planeados [25,26].

La racionalidad encontrada en los programas refleja que los departamentos deben enfocar su esfuerzo al diagnóstico del problema, causas, efectos, necesidades y población objetivo, en los contextos de la región, basándose en evidencia (estudios, investigaciones, estadísticas, etcétera) que fortalezcan, desde lo académico, la percepción en el macroentorno de cada región. El Ministerio de Salud propone una metodología para el análisis de los determinantes sociales de la salud (ASIS), cuyo objetivo principal es "facilitar la caracterización de la situación de salud de la población en los ámbitos municipal, departamental y distrital" [27].

En esta línea, la Universidad Autónoma Nacional de México resalta y presenta una metodología de diagnóstico de situación en salud, que puede retomarse en los departamentos en la planificación de sus programas [28]. De igual forma, la Fundación Kellogs llama la atención sobre fortalecer el planteamiento del problema como punto de partida hacia la "efectividad eventual de los programas" [19], y en la evaluación de las necesidades de salud se resaltan, entre otros, los métodos epidemiológicos, cualitativos y comparativos para describir los problemas de salud de una población [29].

De no existir esta evidencia científica, se invita a las personas implicadas en la toma de decisiones a iniciar estudios orientados al objetivo o en su defecto a utilizar la información hallada en el sistema de monitoreo que posee el programa. De igual forma, es pertinente que los programas consoliden el análisis del diagnóstico particular con una adecuada sustentación científica de la realidad macrocontextual que consolide el diagnóstico en general [9].

El desarrollo del diagnóstico en programas de actividad física permitirá un desarrollo en la modificación de teorías del comportamiento, la medición de variables adicionales en los estudios y el uso de técnicas estadísticas, entre otras, lo que resultará en un mejor uso de la teoría y variables explicativas. Se espera que esto conduzca a una actividad física basada en la evidencia con intervenciones más eficaces [30,31].

Otro aspecto que tener en cuenta dentro de la relevancia y en el que cabe focalizar la atención, es el análisis contextual que se presenta en cada uno de los programas. Se ilustra una descripción geográfica y características climáticas generales, al contrario de las entrevistas, donde los gestores enfatizan en problemáticas como la violencia, las vías de acceso o la cultura propia de la región, que hacen que algunas determinaciones del programa sean modificadas por este tipo de categorías emergentes, representadas en aseveraciones como: “[...] es un departamento con conflicto armado y la fuerza pública lo que quiere es llegar a la población a través de estos programas [...]” (Gestor de Arauca); “[...] En la costa la gente le gusta el baile y eso ha sido como un imán para arrastrar a personas porque ha sido bien difícil llevarlos a una máquina a que hagan cosas verdad” (Gestor del Atlántico); "Putumayo según el DANE el 52\% pertenece el sector rural y el sector rural no es a cinco minutos, sino un sector por el río, muchos kilómetros lejos de los campos urbanos entonces esa es una condición que se debería tener en el departamento del Putumayo" (Gestor del Putumayo).

Estas disposiciones hacen que los actores —entendidos desde la política pública - involucrados en el programa [11] tengan en cuenta estas variables al momento de determinar cobertura, estrategias de intervención, metas, objetivos y disposición de recursos. Solo así el programa tendrá en cuenta la diversidad poblacional y topográfica del país y que es pertinente a la hora de la planificación de cualquier programa social $[32,33,34]$.

Lo anterior se logrará con una continua investigación y revisión de la literatura existente que permita entender las regiones no solamente como un grupo de personas que comparten un lugar geográfico, sino un orden social en el cual los individuos que la conforman tienen en común aspectos como la forma de entender su realidad cotidiana y sus modos de vida. Esta cognición permite diseñar interacciones con una mayor probabilidad de éxito [35]. 
Ha sido fundamental la capacitación de los gestores y los monitores encargados del programa, no solo a partir del insumo de capacitación que tiene Coldeportes para tal fin, sino también gracias a los esfuerzos departamentales por consolidar un grupo de trabajo, pues muchos gestores mencionan lo difícil que resulta encontrar personal capacitado:

“[...] Yo pensaría que el sistema de capacitación que tiene Caldas es bueno, para mí es muy importante que las personas tengan las competencias para dar una clase o una información. Hemos buscado que los muchachos que llegan con poca experiencia en actividades musicalizadas puedan prepararse en esta área y a su vez en los otros temas relacionados con el programa. (Gestora de Caldas)"

"[...] El tema de actividad física ha sido relegado para un pequeño grupo de profesores, ¿qué es lo que pasa? que no podemos tener una cantidad de profesores, [...], porque no los hay, en Cundinamarca [...], hemos enfatizado como equipo de trabajo, en poder capacitar el equipo de trabajo como tal, podernos capacitar internamente. (Gestor de Cundinamarca)"

Este tipo de iniciativas consolidan las desarrolladas por el Lancet Physical Activity Series Working Group, que argumentan la importancia de la capacitación, la formación laboral y el desarrollo intersectorial, enfocados en todas las regiones, con el propósito de fortalecer la investigación, la práctica, la política, la promoción y la educación en el área de la actividad física [32]. Esto fortalece la coherencia del programa y contribuye al sentido de pertenencia por parte de los actores principales.

La mayor fortaleza que tiene el programa es la coherencia interna, reflejada en la jerarquización de los objetivos, planteándose igualmente la correspondencia de estos con la problemática específica del sedentarismo. Los resultados que presentan están mediados por indicadores de proceso, que limitan o no dejan visualizar los avances que los objetivos tienen durante el desarrollo.

Aspectos como el grado de satisfacción, la motivación, la rotación de los usuarios, el grado de felicidad, la empatía con los beneficiarios del programa son ejemplo de algunos indicadores que pueden ser incluidos en la presentación de los resultados y no remitirse al cumplimiento de un determinado número de actividades [36,37].

Ejemplo de algunas intervenciones donde se centran en este tipo de variables se encuentran en programas donde estudian la relación de bienestar psicológico y ejercicio físico $(38,39)$. Y hay quienes concluyen: "El entrenamiento psicológico puede ser más adecuado para las personas mayores frágiles con enfermedades crónicas, que el entrenamiento de actividad física, y puede ayudar a preparar a estas personas para la actividad física" [40].

Lo mencionado rescata la importancia de la propuesta de Marion Herens et al. y otros autores que dirigen la atención a crear un enfoque de evaluación alternativo, donde se perciban nuevos indicadores, métodos y herramientas de un mundo real que comprende múltiples niveles, que deben ser tenidos en cuenta en los diferentes abordajes del diseño de evaluación para la actividad física comunitaria $[41,42]$.

En el desarrollo de las actividades se resaltan los eventos masivos que tiene el programa, convirtiéndose en espacios donde el usuario de forma libre realiza la actividad física, no solo para prevenir enfermedades, sino la oportunidad en mejorar aspectos como la interacción entre las personas pertenecientes a la comunidad, la recuperación y el aprovechamiento de espacio público, mejorando las condiciones medioambientales [43] y la reducción de las desigualdades sociales, entre otras, que pueden ser presentadas en las metas de los programas y que en la actualidad no lo son [44].

Esto se une a los esfuerzos y a la invitación de Lancet Physical Activity Series Working Group de elaborar con urgencia políticas que ataquen la pandemia mundial del sedentarismo y que conviertan a la actividad física no solo en oportunidad en la promoción de la salud, sino en un derecho humano básico [4,32,45,46].

Este tipo de intervención contribuye a dar cumplimiento a cada uno de los objetivos en las diferentes dimensiones prioritarias que presenta el Plan Decenal de Salud Pública 2012-2021 [47], así como del Plan decenal de la recreación, la educación física y la actividad física, para el desarrollo humano, la convivencia y la paz 2009-2019 [48] y El Plan Nacional de Desarrollo 2014-2018. Este último direcciona la salud como 
una de las condiciones básicas para preservar el capital humano, promoviendo hábitos de vida saludable que permitan mitigar la pérdida de años de vida por condiciones no transmisibles [49].

Los eventos masivos son un reflejo del desarrollo intersectorial que tiene el programa, pero su documentación, así como su articulación planificada con otras políticas o programas instaurados en los departamentos muestra algunas deficiencias, y con su modificación puede llegar a pensarse que aumentará la coherencia externa del programa, tal y como lo expone el Comité Ejecutivo de la Serie 2 Actividad Física Lancet [50], siendo esta una de las barreras al progreso de las políticas nacionales sobre actividad física [51].

El programa Hábitos y Estilos de Vida Saludable, de acuerdo con la taxonomía de los programas de actividad física en América [52], está en la tipología 2, sin embargo, la entrevista semiestructurada mostró una variabilidad, fundamentalmente hacia la estrategia comunitaria en el subdominio información y hacia las recomendaciones Task Force, subdominio barreras [52], no evidenciadas en el análisis documental realizado, lo que puede estar mostrando que los formatos utilizados por Coldeportes para recopilar la información no permiten que los monitores y los gestores presenten la particularidad de las actividades, debido a lo estructurado y a la cantidad de formatos que entregar, como fue enunciado por alguno de ellos: "hay demasiados instrumentos, en algún momento dado pensamos que se deja de intervenir, hay que estar más atento a los usuarios" (Gestor del Guainía).

\section{Conclusiones}

La evaluación de estructura en los programas de actividad física es una herramienta eficaz en el mejoramiento del programa Hábitos y Estilos de Vida Saludable, se invita a los actores sociales a que se realice desde la planificación de los programas departamentales, estructurando aún más el documento existente en busca de mayor documentación, y fortaleciendo el trabajo intersectorial que consolide un programa de actividad física para la región, con los lineamientos estipulados por Coldeportes, institución estatal encargada para tal fin.

Lo anterior se logrará gracias a la investigación y el impulso local en lo que se refiere al desarrollo del diagnóstico y a la racionalidad en general de cada uno de los departamentos, así como en el desarrollo de actividades como las que el programa realiza, esto permitirá unir esfuerzos que orienten hacia una política en la prevención de la salud a nivel local.

Se requiere desarrollar protocolos para documentar algunas actividades que los departamentos realizan, pero no quedan registradas. Esto permitirá establecer mayores diferencias entre los departamentos y los efectos que ellas puedan tener en los objetivos trazados.

Las metas que sugieren los diferentes programas deberán tener en cuenta, en próximos desarrollos, las conductas de los usuarios que se presentan en el campo conductual, donde se despliega el programa, empatía, motivación, adherencia, cohesión de grupo, entre otras, unidas a las características contextuales propias de cada grupo y región, como por ejemplo clima, condiciones de seguridad, accesibilidad, iluminación etcétera, invitan y fortalecen las orientaciones de los académicos a diseñar estrategias de evaluación donde se miren los programas en vivo.

Se invita a unificar los criterios que Coldeportes refiere a los departamentos para la ejecución del modelo lógico, pues presentan diferencias en lo relacionado con los indicadores propuestos por el Department for International Development del Reino Unido, y que pudieron intervenir en la evaluación (véase tabla 1).

\section{Agradecimientos}

Artículo de investigación desarrollado durante los años 2015 y 2016 que hace parte del proyecto de investigación Evaluación del componente regular del Programa Nacional de Promoción de la Actividad Física y otros Hábitos Saludables, Colombia, 2014-2015, con el apoyo de la Universidad del Rosario, Escuela de 
Medicina y Ciencias de la Salud, Centro de Estudios en Medición de la Actividad Física (CEMA), Bogotá, Colombia.

\section{Referencias}

1. Coldeportes. Lineamientos y oportunidades de mejora en el desarrollo de la acción de gestores, monitores y líderes del programa de Hábitos y Estilos de Vida Saludable, 2014. Bogotá: Coldeportes; 2014.

2. Hoehner CM. Physical activity interventions in Latin America. A systematic review. Am J Prev Med. 2008 Mar; $34(3): 224-33$.

3. Task Force on community preventive services. Recommendations to increase physical. Am J Prev Med. 2002; 22(4S):67-72.

4. ONU. Declaración Política de la Reunión de Alto Nivel de la Asamblea General sobre la Prevención y el Control de las Enfermedades No Transmisibles. Nueva York: OMS, Asamblea General; 2011. Report No.: A/66/L.1

5. OMS. Estrategia Mundial sobre Regimen Alimentario, Actividad Física y Salud. Ginebra: ONU; 2004.

6. Prieto F. Evaluación del componente regular del Programa Nacional de Promoción de la Actividad Física y Otros Hábitos Saludables, Colombia, 2014-2015 [en prensa]. Bogotá, 2015.

7. Coldeportes. Documento técnico con los contenidos de direccionamiento pedagógico para la promoción de hábitos de vida saludable, con énfasis en alimentación saludable y el fomento de ambientes $100 \%$ libres de humo. Bogotá: Imprenta Nacional; 2011.

8. CDC, U.S. Centers for Disease Control and Prevention - Division of Public Health Systems and Workforce Development, Office of Health Promotion, National Center for Chronic Disease Prevention and Health Promotion. Planeación de Programas (version 2), 2012.

9. Llaneza C. Evaluación del diseño de políticas públicas: propuesta de un modelo integral. Revista del CLAD Reforma y Democracia. 2013; (57):1-18.

10. The World Bank. The road to results: Designing and conducting effective development evaluations. Washington: The World Bank; 2009.

11. Ministerio de Salud y Protección Social, OPS, OMS. Propuesta de herramientas técnicsa para fortalecer el seguimiento y monitoreo de los planes, proyectos y estrategias de las enfermedades no transmisibles. Bogotá: Imprenta Nacional; 2016.

12. Osuna JL, Marquez C. Guía para la evaluación de políticas públicas. Sevilla, España: Instituto de Desarrollo Regional: s.f.

13. Mahecha M, Rodríguez V, Andrade D, Araujo T. Evaluation of a physical activity promotion program: The example of Agita Sao Paulo. Elsevier; 2006: p. 301-11.

14. The Joanna Briggss Institute. Joanna Briggs Institute reviewers' manual. En: Manual. JBIR. Reviewers' manual. Adelaida (Carolina del Sur): The Joanna Briggss Institute; 2014. p. 183.

15. Coneval. Modelo de términos de referencia para la evaluación en materia de diseño. Mexico: Coneval; 2015.

16. Department for International Development (DFID). Guidance on using the revised logical framework. How to note. DFID; 2011.p. 135.

17. Coldeportes. Guía Para la presentación de proyectos de hábitos y estilos de vida saludable [documento interno]. 2014.

18. Coldeportes. Guía para la presentación de proyectos de hábitos y estilos de vida saludable [documento interno]. 2015.

19. W. K. Kellogg Foundation. Guía de desarrollo de modelos lógicos. Michigan: W. K. Kellogg Foundation; 2001.

20. Mayan MJ. Una introducción a los métodos cualitativos: módulo de entrenamiento para estudiantes y profesionales. Toronto, Canadá: International Institute for Qualitative Methodology; 2001.

21. Alvarez J. Cómo hacer evaluación cualitativa. Barcelona: Paidós; 2003. 
22. Castillo E. El rigor metodológico en la investigación cualitativa. Colombia Médica. 2003; 34(3):164-7.

23. Ministerio de Salud y Protección Social, Instituto Colombiano de Bienestar Familiar. Encuesta Nacional de la Situación Nutricional en Colombia 2010 (Ensin). Bogotá: Imprenta Nacional; 2011.

24. Ministerio de Salud, INS. Herramientas para la gestión del riesgo de las enfermedades cardiovasculares y diabetes. Bogotá: Imprenta Nacional; 2006.

25. World Health Organization Europe. Evaluation in health promotion. Principles and Perspectives. Copenhague: WHO; 2001.

26. Matamoros GO. Manual de analisis y diseño de políticas públicas. Bogotá: Universidad Externado de Colombia; 2013.

27. Ministerio de Salud y Proteccion Social. Guía conceptual y metodológica para la construcción del Análisis de Situación en Salud (ASIS) de las entidades territoriales. Bogotá: Imprenta Nacional; 2014.

28. Universidad Nacional Autonoma de México. Manual de prácticas de medicina preventiva y salud pública. Mexico D. F.: Universidad Nacional Autonoma de México; 2008.

29. Wright J. Development and importance of health needs assessment. PubMed Central (PMC); 1998. p. 37-40.

30. Bauman A. Toward a better understanding of the influences on physical activity: the role of determinants, correlates, causal variables, mediators, moderators, and confounders. American Journal of Preventive Medicine. 2002; 23(2):5-14.

31. Cabrera G. Actividad física y etapas de cambio comportamental en Bogotá. Revista Colombia Médica. 2004; 35(2):82-6.

32. Kohl HW, et al. The pandemic of physical inactivity: global action for for public health. The Lancet. 2012; 380(9838):294-305.

33. Verde L. Evaluating the relevance, generalization, and applicability of research issues in external validation and translation methodology. Evaluation \& The Health Professions. 2006; 29(1):126-53.

34. Gómez L. Revisión sistemática de intervenciones comunitarias sobre actividad física en grupos específicos. Colombia Médica. 2002; 33(4):162-70.

35. Robledo-Martínez R. Características socioculturales de la actividad física en tres regiones de Colombia. Salud Pública. 2006; 8(Supl. 2):13-27.

36. Castro J. Aspectos asociados a la actividad física en el tiempo libre en la población adulta de un municipio antioqueño. Salud Pública. 2008; 10(5):679-90.

37. Bohórquez M. Felicidad y actividad física en personas mayores. Escritos de Psicología. 2013; 6(2):6-12.

38. Campos J. Efectos de un programa de ejercicio físico sobre el bienestar psicológico de mujeres mayores de 55 años. Psicología del Deporte. 2003; 12(1):7-26.

39. Zafra O. Variables sociodemográficas, ejercicio físico, ansiedad y depresión en mujeres: un estudio correlacional. Revista Internacional de Medicina y Ciencias de la Actividad Física y el Deporte. 2008; 8(31):224-43.

40. Van Heuvelen MJ. Psychological and physical activity training for older persons: who does not attend? Gerontology. 2006; 52(6):366-75.

41. Herens M, Wagemakers A, Vaandrager L, Van Ophem J, Koelen M. Evaluation design for community-based physical activity programs for socially disadvantaged groups: Communities on the move. JMIR Research Protocols. 2013; 2(1):1-14.

42. Rabin B. Methodologic challenges in disseminating evidence-based interventions to promote physical activity. American Journal of Preventive Medicine. 2006; 31(4):24-34.

43. Kahn EB. The effectiveness of interventions to increase physical activity. A systematic review. Elsevier Science; 2002.

44. Ball K, et al. Addressing the social determinants of inequities in physical activity and related health outcomes. Health Promotion International. 2015: 30(Supl. 2):ii8-ii19.

45. Republica de Colombia. Constitución Política de Colombia. Bogotá: Imprenta Nacional; 1991.

46. Vidarte J. Actividad física: estrategia de promoción de salud. Hacia la Promoción de salud. 2011; 16(1):202-18. 
47. Ministerio de Salud y Protección Social, Republica de Colombia. Plan Decenal de Salud Pública, PDSP, 2012-2021. Bogotá: Imprenta Nacional; 2013.

48. Ministerio de Cultura, Instituto Colombiano del Deporte. Plan decenal de la recreación, la educación física y la actividad física, para el desarrollo humano, la convivencia y la paz 2009-2019 Bogotá: Imprenta nacional; 2009.

49. Departamento Nacional de Planeación. Colombia. Plan Nacional de Desarrollo 2014-2018: Todos por un nuevo país, tomo 1. Bogotá: Imprenta Nacional de Colombia; 2015.

50. Sallis J. Physical activity 2016: Progress and challenges. Progress in physical activity over the Olympic quadrennium. The Lancet. 2016; 388(10051):1325-36.

51. Mena-Bejarano B. Análisis de experiencias en la promoción de actividad física. Salud Pública. 2006: 8(Supl. 2):42-56.

52. Rodríguez A. Taxonomía de los programas comunitarios de actividad física en América: una revisión mixta [tesis de investigación en la Maestría en Actividad Física y Salud]. Bogotá: Universidad del Rosario; 2016.

\section{Notas}

* Artículo de investigación

Financiamiento de la investigación

Fuente: Universidad del Rosario, Escuela de Medicina y Ciencias de la Salud, Centro de Estudios en Medición de la Actividad Física (CEMA)

Licencia Creative Commons CC BY 4.0

Como citar este artículo: Torres Moya OR, Prieto Alvarado FE. Evaluación de la estructura del componente regular de los programas departamentales de hábitos y estilos de vida saludable, Colombia, 2014- 2015. Rev Gerenc Polít Salud. 2017; 17(34): 1-18. https://doi.org/10.11144/Javeriana.rgps17-34.esrc 